\title{
THE ROLE OF COMMUNITY PHARMACIST IN ASSESSMENT AND MANAGEMENT OF ATOPIC DERMATITIS
}

\author{
Jelena D. Milosavljević ${ }^{1}$, Milena D. Nikolić ${ }^{2}$ \\ ${ }^{1}$ Community pharmacies , Kruševac, Republic of Serbia \\ jelenamilospharm@gmail.com \\ ${ }^{2}$ College of applied sciences in technical studies and technology, Kruševac, Republic of Serbia \\ milena.nikolic.1987@gmail.com
}

Original Scientific Paper doi:10.5937/jouproman6-16223

\begin{abstract}
This issue is a review of treatment for atopic dermatitis - chronic, recurring inflammatory disorder of the skin. For a large number of patients community pharmacist is the most accessible healthcare professional. Pharmacist can give adequate advice about nature of disease and treatment options.

This article aims to analyze the therapeutic outcome of 30 patients who asked for advice from a community pharmacist. Subjects were patients with skin problems that have been targeted by eczema. Pharmacist used a standardized questionnaire in order to collect data. The results shows there is a positive feedback between a favorable therapeutic outcome and a given pharmacist advice. Material and work methods are described and some conclusions are presented in the text bellow.
\end{abstract}

Keywords: eczema, assessment, emollients, topical steroids

\section{Introduction}

Atopic dermatitis is very common chronic skin disease, affecting $10-20 \%$ of children and $2-10 \%$ of adults.

There are two phases in this skin condition - exacerbation and remission.

In acute phase, skin is very dry, irritated, itcy, erythematous, and sometimes exudated.

"The subsequent chronic inflammatory phase is characterized by scaling, excoriations, skin thickening and enhancement also known as lichenification.
Atopic eczema affects mainly the flexor surfaces of the elbows and knees, as well as the face and neck, although patterns of localization can differ depending on the age of the patient. In babies and small children eczema is common on the checks, forehead and extensor surface of the limbs" (www.medicinesni.com)

Atopic dermatitis is diagnosed clinically.

What are the aims of the treatment?

"The aims of the treatment are to heal skin and to keep it healthy, prevent flare-ups and treat symptoms as soon as they occur and so improve quality of life" (www.medicinesni.com)

"There is a NICE clinical guideline concerning the management of eczema in children from birth up to the age of 12 years.

The scope of the NICE guideline is limited to children under 12 years. The recommendations can be extrapolated to the management of older children and adults with atopic eczema" (www.medicinesni.com), with additional information added.

The flare of atopic eczema NICE defines as "an increase in clinical severity (redness or itching) of the condition". 
Very important in this condition is to recognize and to treat flare-ups at the very beginning. The duty of healthcare professionals is to educate patients about nature of this skin condition so they could recognize flare-ups at the beginning.

\section{Treatment}

For most of the patients affected by atopic dermatitis the care is taken by primary care professionals. They should be referred to specialist-dermatologist "if the condition is severe and has not responded to appropriate therapy" (www.medicineni.com).

For many patients affected to atopic dermatitis, community pharmacist is the most accessible healthcare professional. Pharmacist can give advice about nature of disease, aims of treatment, duration of treatment, appropriate use of therapeutic agents, importance of compliance and treatment options.

\subsection{Emollients}

Emollients "are almost universally recommended as first-line treatment. Emollients have soothing effect on itching and soreness" (www.medicinesni.com), they may have an anti-inflammatory effects. Technically, emollient is "being something that smoothes and softens the skin, usually via occlusion. The correct emollient is the one that the patient will use. Adherence to emollient treatment is the key to successful therapy for atopic eczema." (www.medicinesni.com). Patient should be advised to use them "liberally and frequently, even when the skin appears improved or is clear" (www.medicinesni.com). Patients should be supplied with big dosage pack for use at home, and smaller dosage pack for use at work, at school, while traveling. Avoidance of using soaps, detergents and bubble bath is general recommendation.

"Patients may require more than one emollient product, depending on lifestyle, time of day, seasonal factors, cite of application or disease severity. Emollients have a steroid-sparing effect, and should be supplied in a 10:1 ratio of emollient to steroid in order to achieve the full benefit. There should be a time interval of at least 30 minutes ( $1 \mathrm{~h}$ for tacrolimus) between the application of topical flare treatments (topical corticosteroids, topical immunomodulators) and emollient." (Moncrief et al. Use of emollients in dryskin conditions: consensus statement. Clinical and Experimental Dermatology, 2013). "Ointments are preferable for dry skin because they are more effective than creams, but creams are preferable on red, inflamed skin because the evaporation of water-based products cools the skin" (www.medicines.ni).

Pump-dispensers are recommended if large amounts of emollients should be used for large areas of skin.

\subsection{Topical corticosteroids}

"Many patients with atopic eczema need to use topical corticosteroid preparations intermittently when the condition flares and a few patients need to use them long-term. Topical corticosteroids are the first-line treatment for flare - ups of atopic dermatitis. They do not cure the condition, but they will control it via antiinflammatory and immunosuppressive effects. They reduce inflammation and relieve itching. Evidence from controlled trials for effectiveness of this group of drugs is limited. Clinical experience has shown that topical steroids improve eczema over a two to four week period. Initial improvement should be seen within $3-7$ days of starting steroids" (www.medicinesni.com). 
There are several sources to categorize topical corticosteroids. According to British National Formulary, there are five groups of topical steroids: mild, moderately potent, potent and very potent. "The potency is measured by its ability to cause vasoconstriction, rather than its clinical effectiveness. Very potent corticosteroids are up to 600 times as potent as mild topical corticosteroids. The choice of topical corticosteroid is based on potency of preparation and the site and severity of the condition." (www.medicinesni.com)

The adverse effects of topical steroids are not difficult for identification. "Side - effects generally only occur following the incorrect use of potent steroids over a long period of time.

Local side-effects include:

- Transient burning or stinging is common and may necessitate changing the product.

- Spread and worsening of untreated infection.

- The skin can become thin and easily bruised. It is common in the skin of the elbow creases and behind the knees.

- Irreverisible striae atrophicae-prolonged or excessive use of potent steroids causes the dermis lose its elasticity and stretch marks (striae) to appear, which are permanent.

- Rebound flare- if topical steroids are stopped abruptly

- Glaucoma following application of topical steroids on the eyelid region

When patients are using topical steroids in adequate way, systemic adverse effects are very rare; they can include adrenal suppression that can result in symptoms of Cushing's syndrome and growth retardation in children" (www.medicinesni.com).

Topical steroids-how should patients use them?
Topical corticosteroids are usually used once-daily in treatment of atopic dermatitis. Twice-daily application could be included if symptoms are not resolved and then stepping down again to once-daily application.

Topical steroids should be used 3 7 days for management of flares. Sometimes, courses of $7-14$ days are necessary. If symptoms are not resolved within 7-14 days, the secondary bacterial or viral infection may be present. Topical corticosteroids are only used to treat affected surfaces and treatment should be continued for 48 hours after symptoms resolve. The recommendation is if moderate potency product is used, to step down to low potency for a few days.

\subsection{Topical calcineurin inhibitors}

In this issue, the emphasis is on the first-line therapeutic agents for management atopic dermatitis - emollients and topical corticosteroids. Therefore, the agents for second - line treatment are reviewed just informative.

In Serbia one agent from group of topical calcineurin inhibitors is registered for treatment of atopic eczema: pimecrolimus (Elidel cream). In other countries tacrolimus

(Protopic ointment) is also available.

"Topical tacrolimus is an option for the second-line treatment of moderate to severe atopic eczema in adults and children aged 2 years and older.

Topical pimecrolimus is an option for the second - line treatment of moderate atopic eczema on the the face and neck in children aged 2 - 16 years." (www.medicinesni.com) 
Treatment with tacrolimus and pimecrolimus should be carried out under the supervision of a specialistdermatologist. Patient should be informed about the potential risk and benefits of treatment.

"Unlike topical corticosteroids, tacrolimus and pimecrolimus do NOT interfere with collagen synthesis or induce skin atrophy. This could be a major advantage of long-term use and for use on the face and neck" (www.medicinesni.com).

\subsection{Systemic therapy of atopic eczema}

Sometimes, when moderate to severe atopic eczema is present, it could not me managed only with topical steroids and emollients.

In those cases, cyclosporin and azathioprine are used. Those agents are used only in a secondary-care institutions.

\subsection{Antihistamines}

In the treatment of eczema, antihistamines are recommended when itching is the dominant inconvenience.

In the case of a patient having a problem with insomnia, a sedative antihistamine may be included.

\subsection{Oral corticosteroids}

If large areas of skin are involved (more than $40 \%$ of surface area), oral corticosteroids are used, in acute flares, but for short - time period.

\subsection{Phototherapy}

This treatment option is exclusively carried out under the supervision of a specialist - dermatologist.

\subsection{Bandages, medicated dressings and wet wrap therapy}

Medicated dressings and wet wrap therapy are treatment options for lichenified areas in children and for treatment of flares in short- time period.

\subsection{Infection and the role of antimicrobials}

In some cases, a bacterial infection may further aggravate the condition of the patient with eczema.

The most commonly present are the infections with Staphylococcus aureus and occasionally with Streptococcus pyogenes.

In such cases, when the symptoms are recognized, antimicrobials could be used. They are available both as topical and systemic antimicrobials.

It is important to mention the patient to purchase new supplies of topical medications after completion of the treatment of the infected area.

During treatment with antimicrobials, the usual therapy (emollients, topical steroids ) continues.

\section{Object and purpose of the investigation}

In this article authors tried to investigate whether a simple pharmacist intervention delivered within the scope of everyday practice could promote management of atopic dermatitis. Patients with skin problems that have been targeted by eczema were questioned.

\section{Material and methods of work}

The investigation was conducted in one community pharmacy. Two pharmacists have participated investigation. It took a month. Thirty patients were questioned. The authors used Pharmacist Assessment Document (www.medsask.usask.ca). 
The pharmacist first took general information from patient - date of birth, gender, is pregnany or lactation present, medical history, drug history, drug allergies.

The next step was taking patient history. The patients were asked if there was family history of atopic dermatitis. In order to eliminate the suspicion of another skin problems (scabies, pediculosis e.g.) and differential assessment be done patient was asked if he has recently been in contact with person with similar skin conditon. If answer was positive, pharmacist referred patient to dermatologist. If patient has recently been in contact with unknown plant or exposed to chemicals pharmacist should refer him to dermatologist. If there was a suspicion that skin condition is an adverse drug reaction, patient should be reffered. Patients with large areas of skin involved (more than 30 $\%$ of surface area), moderate or severe lesions on face, signs or symptoms of infection should be referred.

After differential assessment was done, pharmacist gave an adice for treatment to patient. The advice consists in administering non - pharmacologic measures: trigger avoidance, use of emollients, moisurizers. Topical corticosteroid is administered according to the guideline. In treatment eczema in infants - low potency corticosteroid for all areas of skin involved. For older children and adults - low potency corticosteroid should be included in treatment areas on face and skin folds. For older children and adults, low to moderate potency steroid should be administreted in treating areas on body, limbs and scalp.

Patients are said to expect relief of symptoms within 7 days, if no response or symptoms worsen to contact pharmacist. They should continue corticosteroid use for 48 hours after symptoms resolve. Subjects were scheduled a follow - up after a week.

\section{Concluding observations}

Of 30 patients tested 24 reported to the follow -up after a week $(80 \%)$ and finished intervention. The average age of the respondents was 38,5 years. There were 18 respondents female and 12 male.

On the first review many patients said that they are not getting enough information during visiting doctor or they never see them for such problem. The source of information is internet for majority of them.

Three patients reported bronchial asthma and four reported allergic rhinitis in childhood. To most of them (21 of 30), previous diagnose of atopic dermatitis was done. One patient reported to have been in contact with chemical- motor oil. He has previously been diagnosed with hand eczema by his dermatologist. His condition becomes worse on cold weather and in contact with chemicals. The advice given to him was to avoid triggers, use emollients and treat skin changes with topical steroid of low potency for seven days. One patient reported skin changes in stomach area. She started to use new drug - "lisinopril tablets $10 \mathrm{mg}$ ". Pharmacist recommended to stop use medicine and referred patient to specialist. There were no patients reported signs or symptoms of infections. There were no patients with large area of skin involved.

As far as the outcome of the treatment is concerned the results are cited bellow:

- 19 patients (of 24 who came on followup) reported that the symptoms resolved. They were advised to stop corticosteroid treatment and to continue regular use of emollients.

- 4 patients (of 24) reported that the symptoms improved. They were counseled to continue corticosteroid treatment for up to another 7 days. If symptoms are not resolved within 14 days treatment to contact his pharmacist. 
- 1 patient reported intolerability with chosen formulation. It was too greasy, patient did not use it regularly. New preparation was included and repeated follow - up within 7 days. After 7 days treatment with another preparation, he reported that symptoms resolved.

Pharmacists found that some patients who have previously been diagnosed with atopic dermatitis and already have used emollients, apply them ineffectively- inadequate amount of emollients, inadequate accuracy. This gap of knowledge and understanding leaded to non-compliance and had an impact on disease outcome. The emphasis was on giving an adequate advice about emollients, whose are in pharmacist competency. Most patients said the pharmacist intervention was quite helpful. Emollient creams and baths are recommended as first-line treatment and effective use may be steroidsparing.

When we are talking about use of topical steroids, the emphasis was on giving an appropriate advice about benefits of treatment, which outweigh adverse effects when they are applied to adequately. Patients were encouraged to use them not "sparingly"; the advice of "finger-tip-unit" for better understanding of the amount of preparation the patient needs to use is given.

Patient education about nature of this skin condition; better understanding of non-pharmacologic treatment: general measures, use of emollients, trigger avoidance improves adherence and quality of live, in which community pharmacist can have an important role. The main advantages the patients listed was the availability of information from community pharmacist and the shortening of time to solve the problem. certainly is not adequate sample for giving representative conclusions, but confirmed literature data the authors had.

\section{References}

[1] Bewley, A., (2009). Changing the way we advise patients on use of topical steroids. Prescriber, 20, 44-48

[2] British Medical Association - Royal Pharmaceutical Society of Great Britain, London (2009), British National Formulary, BNF,58, 2009

[3] Ilić K., Bjekić M. (2011). Lokalni kortikosteroidni preparati u dermatologiji I praksa izdavanja, Arhiv za farmaciju, 3, $279-287$

[4] Moncrieff, G., M.Cork, S. Lawton, S.Kokiet, C. Daly, and C. Clark (2013). Use of emollients in dryskin condition: consensus statement, Clinical and Experimental Dermatology,

[5] National Institute for Health and Excellence (2007), Management of atopic eczema in children from birth up to the age of 12 years, Clinical Guideline 57

[6] National Prescribing Centre (1998). The use of emollients in dry skin condition, MeRec Bulletin, 10, 21-24

[7] Oakley A., (1997, updated 01.2016.). Topical steroid, Topics A-Z, www.dermnetnz.org.org

[8] Saeki, H. et al, (2009). Guidelines for management of atopic dermatitis, Japanese Dermatological Association, The Journal of Dermatology, 10, 563-577

[9] University of Saskatchewan, medSask Guideline, (2017). Atopic Dermatitis, www.medsask.usask.ca.professional

[10] COMPASS Notes (2016). Therapeutic Notes on the Primary Care Pharmacological Management of Atopic Eczema. www.medicinesni.com

This small group of patients which was questioned in this investigation 